\title{
Classification of Two Genera of 32-Dimensional Lattices of Rank 8 over the Hurwitz Order
}

\author{
Christine Bachoc and Gabriele Nebe
}

\section{CONTENTS}

1. Introduction

2. The Mass Formulas

3. Some Notation

4. Results for Rank 1 to 7

5. The Lattices of Rank 8

References
Nebe's research was supported by the Deutsche Forschungsgemeinschaft.
A generalization of Kneser's neighboring method allows us to classify two interesting genera at the same time. The new method is used to determine the genus of Hermitian unimodular lattices of rank 8 over the Hurwitz order $\mathfrak{M}$ and the genus of those $\mathfrak{M}$-lattices corresponding to unimodular $\mathbb{Z}$-lattices.

\section{INTRODUCTION}

Kneser's neighboring method [Kneser 1957] has extensively been used to construct all lattices in a given genus. Essentially one starts with one lattice in the genus and computes its neighbors as overlattices of certain maximal sublattices. So one really classifies two genera of lattices, being only interested in one. In this paper we generalize this method, replacing the maximal sublattices by sublattices of larger index in a more interesting genus. The resulting graph in each genus, which factors over a bipartite graph connecting the two genera (compare Proposition 2.6), contains the original neighborhood graph and hence is connected. We apply this method to determine the genus of unimodular lattices of rank 8 over the Hurwitz order $\mathfrak{M}$, as well as the one consisting of $\mathfrak{P}$-modular M-lattices (compare Definition 2.1). The latter classification was proposed in [Quebbemann 1984], where a mass formula for this genus is developed. There are only 11 such lattices, 8 of which are indecomposable. Four of these lattices are extremal in the sense that they do not contain vectors of length 2 , and give rise to 3 non-isometric extremal even unimodular $\mathbb{Z}$-lattices of dimension 32 . There are $24 \mathfrak{M}$-unimodular lattices of rank 8, 15 of which are indecomposable. All $24 \mathfrak{M}$-unimodular lattices 
contain Hermitian roots, so no extremal 2-modular $\mathbb{Z}$-lattice in the sense of [Quebbemann 1995] of dimension 32 has a structure as an $\mathfrak{M}$-unimodular lattice, which answers a question raised in [Bachoc 1995].

The paper is organized as follows. Section 2 presents the main ideas. They also allow to compute the mass of one genus, once the one of the other genus is known. The notation concerning the lattices is fixed in Section 3. Section 4 is dedicated to the application of the new method to $\mathfrak{M -}$ lattices of rank at most 7 , and Section 4 presents the results for rank 8. Gram matrices and generators for the Hermitian automorphism groups of the indecomposable lattices are available at http:// www.research.att.com/ njas/lattices.

\section{THE MASS FORMULAS}

Let $\mathfrak{Q}$ be the quaternion algebra with center $\mathbb{Q}$, ramified at 2 and $\infty$. Let $\mathfrak{M}$ a maximal order in $\mathfrak{Q}$ and let $\mathfrak{P}=(1+i) \mathfrak{M}$ the two-sided maximal ideal of $\mathfrak{M}$ containing $2 \mathfrak{M}$.

Definition 2.1. Let $V$ be a left $\mathfrak{Q}$-vector space, $h$ : $V \times V \rightarrow \mathfrak{Q}$ a positive definite Hermitian form with respect to the canonical involution of $\mathfrak{Q}$, and $L$ an M-lattice in $V$.

(i) $L$ is called even if $h(x, x)$ is even for all $x \in L$.

(ii) The Hermitian dual lattice $L^{*}$ of $L$ is defined as

$$
L^{*}:=\{x \in V: h(x, l) \in \mathfrak{M} \text { for all } l \in L\} .
$$

(iii) $L$ is called unimodular if $L^{*}=L$.

(iv) $L$ is called $\mathfrak{P}$-modular if $\mathfrak{P} L^{*}=L$.

(v) $L$ is called almost $\mathfrak{P}$-modular if $L \subseteq \mathfrak{P} L^{*}$, and $\mathfrak{P} L^{*} / L \cong \mathfrak{M} / \mathfrak{P}$.

(vi) The Hermitian automorphism group of $L$ is the subgroup $U(L)$ of $\mathrm{GL}(V)$ consisting of $g$ such that $L g=L$ and $h(x g, y g)=h(x, y)$ for all $x, y \in V$.

If $L$ is an $\mathfrak{M}$-lattice such that $L \subseteq \mathfrak{P} L^{*}$, then the values of the Hermitian form on $L$ lie in $\mathfrak{P}$, that is, $h(x, y) \in \mathfrak{P}$ for all $x, y \in L$. Especially $h(x, x)$ lies in $\mathfrak{P} \cap \mathbb{Q}=2 \mathbb{Z}$ for all $x \in L$. Therefore $L$ becomes an even integral lattice with respect to the symmetric bilinear form $(x, y):=\frac{1}{2} \operatorname{Tr}(h(x, y))$, where $\operatorname{Tr}$ is the reduced trace of $\mathfrak{Q}$.

In particular, if $L$ is a $\mathfrak{P}$-modular $\mathfrak{M}$-lattice of rank $n$, this construction yields an even $\mathbb{Z}$-unimodular lattice of dimension $4 n$. So $\mathfrak{P}$-modular lattices do not exist if $n$ is odd. The same construction applied to an almost $\mathfrak{P}$-modular lattice yields a $\mathbb{Z}$-lattice of determinant $2^{2}$. Since $\mathfrak{M}$ itself is an $\mathfrak{M}$-unimodular lattice, there are $\mathfrak{M}$-unimodular lattices of arbitrary rank $n$. Since the different $\mathfrak{P}$ of $\mathfrak{M}$ is a principal ideal, these lattices give rise to 2 -modular integral lattices of dimension $4 n$ with respect to $(x, y)=\operatorname{Tr}(h(x, y))$.

Proposition 2.2. Let $L$ be an $\mathfrak{M}$-lattice with respect to the Hermitian form $h$.

(i) If $L$ is $\mathfrak{M - u n i m o d u l a r , ~ t h e n ~} h$ induces a nondegenerate Hermitian form

$$
\bar{h}: L / \mathfrak{P} L \times L / \mathfrak{P} L \rightarrow \mathfrak{M} / \mathfrak{P} \cong \mathbb{F}_{4}
$$

defined by $\bar{h}(\bar{x}, \bar{y})=\overline{h(x, y)}$ for all $x, y \in L$.

(ii) If $2 L^{*} \subseteq L \subseteq \mathfrak{P} L^{*}$, then $L / 2 L^{*}$ is a nondegenerate symplectic vector space over $\mathfrak{M} / \mathfrak{P} \cong \mathbb{F}_{4}$ with respect to the form

$$
\varphi: L / 2 L^{*} \times L / 2 L^{*} \rightarrow \mathfrak{M} / \mathfrak{P} \cong \mathbb{F}_{4}
$$

given by $\varphi(\bar{x}, \bar{y})=\overline{\frac{1}{2} h(x, y)(1+i)}$ for all $x, y \in$ $L$.

Proof. (i) The form $\bar{h}$ clearly inherits the property to be Hermitian from the form $h$. To see the nondegeneracy choose $x \in L$ with $h(x, y) \in \mathfrak{P}$ for all $y \in L$. Then $\frac{1}{1+i} x \in L^{*}$ and therefore $x \in \mathfrak{P} L$, because $L$ is $\mathfrak{M}$-unimodular.

(ii) For $x, y \in L$, one has $h(x, y) \in \mathfrak{P}$, so

$$
\frac{1}{2} h(x, y)(1+i) \in \mathfrak{M}
$$

Therefore $\varphi$ is well defined. Since $h(x, x) \in 2 \mathfrak{M}$, all vectors are isotropic.

To see the nondegeneracy of $\varphi$ let $x \in L$ with $\frac{1}{2} h(x, y)(1+i) \in \mathcal{P}$ for all $y \in L$. Then $h(x, y) \in$ $2 \mathfrak{M}$ and therefore $x \in 2 L^{*}$. 
The form $\varphi$ is clearly linear in the first variable. To prove the linearity in the second argument let $\rho: \mathfrak{Q} \rightarrow \mathfrak{Q}$ denote the canonical involution and choose $x, y \in L^{*}, b \in \mathfrak{M}$. Since both, the canonical involution $\rho$ and conjugation by $(1+i)$ induce the Frobenius automorphism on $\mathfrak{M} / \mathfrak{P}$, one gets

$$
\begin{aligned}
\varphi(\bar{x}, \overline{b y}) & =\overline{\frac{1}{2} h(x, y) \rho(b)(1+i)}=\overline{\frac{1}{2} h(x, y)(1+i) b} \\
& =\varphi(\bar{x}, \bar{y}) \bar{b}=\bar{b} \varphi(\bar{x}, \bar{y}) .
\end{aligned}
$$

Hence $\varphi$ is bilinear.

The main idea of the method to classify both, the $\mathfrak{M}$-unimodular and the (almost) $\mathfrak{P}$-modular lattices of a given rank is the following observation.

Proposition 2.3. (i) Let $M$ be an $\mathfrak{M}$-unimodular lattice of rank $n$. If $n$ is even, the $\mathfrak{P}$-modular lattices contained in $M$ are the full preimages of the maximal isotropic subspaces of the Hermitian $\mathbb{F}_{4}$ vector space $M / \mathfrak{P} M$. If $n$ is odd, the almost $\mathfrak{P}$-modular lattices contained in $M$ are the full preimages of the maximal isotropic subspaces of the Hermitian $\mathbb{F}_{4}$ vector space $M / \mathfrak{P} M$.

(ii) Let $L$ be an $\mathfrak{M}$-lattice of rank $n$. If $n$ is even, assume that $L$ is $\mathfrak{P}$-modular and if $n$ is odd, assume that $L$ is almost $\mathfrak{P}$-modular. The $\mathfrak{M}$ unimodular lattices containing $L$ are of the form $\mathfrak{P}^{-1} N$, where $N$ is the full preimage of a maximal isotropic subspace of the symplectic $\mathbb{F}_{4}$ vector space $L / 2 L^{*}$ of dimension $\operatorname{dim}_{\mathbb{F}_{4}}\left(L / 2 L^{*}\right)=$ $2 \cdot\left[\frac{n}{2}\right]$.

Proof. (i) Let $L$ be a $\mathfrak{M}$-lattice corresponding to a maximal isotropic subspace of $M / \mathfrak{P} M$. Then $h(L, L) \subseteq \mathfrak{P}$ shows that $L \subseteq \mathfrak{P} L^{*}$. The index can be seen from the dimension of this subspace. Conversely let $L$ be a (almost) $\mathfrak{P}$-modular lattice contained in $M$. Then $L \subseteq L+\mathfrak{P} M \subseteq \mathfrak{P} L^{*}$. Now either $L=\mathfrak{P} L^{*}$ and clearly $\mathfrak{P} M \subseteq L$ or $\mathfrak{P} L^{*} / L \cong \mathfrak{M} / \mathfrak{P}$ and one has equality in one of the two inclusions above. Equality in the first inclusion directly implies $\mathfrak{P} M \subseteq L$. Equality in the second inclusion yields $\mathfrak{P} L^{*} \subseteq M=M^{*} \subseteq \mathfrak{P}^{-1} L$. Clearly the image of $L$ in $M / \mathfrak{P} M$ is maximal isotropic.

Part (ii) is analogous.
To prove the completeness of the lists of isometry classes of $\mathfrak{M}$-unimodular and (almost) $\mathfrak{P}$-modular lattices we use the following mass formula, developed in [Hashimoto 1980]:

Let $M_{1}, \ldots, M_{h}$ be the Hermitian isometry classes of unimodular $\mathfrak{M}$-lattices of rank $n$. Then

$$
\sum_{i=1}^{h} \frac{1}{\left|U\left(M_{i}\right)\right|}=\prod_{i=1}^{n} \frac{\left(2^{i}+(-1)^{i}\right) B_{i}}{4 i}
$$

where $B_{i}$ is the $i$-th Bernoulli number.

Using this formula, a mass formula for the genus of (almost) $\mathfrak{P}$-modular lattices can be easily derived by a counting argument, which the second author learned from B. B. Venkov:

Proposition 2.4. Let $M_{1}, \ldots, M_{h}$ be representatives of the isometry classes of unimodular $\mathfrak{M}$-lattices of rank $n$. Let $L_{1}, \ldots, L_{s}$ be representatives of the isometry classes of $\mathfrak{P}$-modular (if $n$ is even) or almost $\mathfrak{P}$-modular (if $n$ is odd) $\mathfrak{M}$-lattices. Let $c_{1}$ denote the number of maximal isotropic subspaces of the Hermitian $\mathbb{F}_{4}$ vector space of dimension $n$ and $c_{2}$ denote the number of maximal isotropic subspaces of the symplectic $\mathbb{F}_{4}$ vector space of dimension $2 \cdot\left[\frac{n}{2}\right]$. Then

$$
\sum_{j=1}^{s} \frac{1}{\left|U\left(L_{j}\right)\right|}=\frac{c_{1}}{c_{2}} \sum_{i=1}^{h} \frac{1}{\left|U\left(M_{i}\right)\right|} .
$$

Proof. For $1 \leq i \leq h$ and $1 \leq j \leq s$ let

$$
a_{i j}:=\mid\left\{L \leq M_{i}: L \text { is isometric to } L_{j}\right\} \mid
$$

and

$$
b_{j i}:=\mid\left\{\mathfrak{P} M \leq L_{j}: M \text { is isometric to } M_{i}\right\} \mid .
$$

By Proposition 2.3 one has $\sum_{j=1}^{s} a_{i j}=c_{1}$ and $\sum_{i=1}^{h} b_{j i}=c_{2}$.

Let $\varphi$ be a unitary mapping with $\varphi\left(L_{j}\right) \leq M_{i}$. Then $\mathfrak{P} M_{i} \leq \varphi\left(L_{j}\right)$ and hence $\mathfrak{P} \varphi^{-1}\left(M_{i}\right) \leq L_{j}$. So the number of unitary embeddings of $L_{j}$ into $M_{i}$ equals the number of unitary embeddings of $\mathfrak{P} M_{i}$ into $L_{j}$. Moreover, if $\varphi^{\prime}$ is a further unitary 
embedding of $L_{j}$ into $M_{i}$, with $\varphi\left(L_{j}\right)=\varphi^{\prime}\left(L_{j}\right)$, then $\varphi^{\prime} \varphi^{-1} \in U\left(L_{j}\right)$. Therefore one has

$$
a_{i j}\left|U\left(L_{j}\right)\right|=b_{j i}\left|U\left(M_{i}\right)\right| .
$$

Hence

$$
\begin{aligned}
c_{1} \sum_{i=1}^{h} \frac{1}{\left|U\left(M_{i}\right)\right|} & =\sum_{j=1}^{s} \sum_{i=1}^{h} a_{i j} \frac{1}{\left|U\left(M_{i}\right)\right|} \\
& =\sum_{j=1}^{s} \sum_{i=1}^{h} b_{j i} \frac{1}{\left|U\left(L_{j}\right)\right|} \\
& =c_{2} \sum_{j=1}^{s} \frac{1}{\left|U\left(L_{j}\right)\right|} .
\end{aligned}
$$

Note that an analogous proof may be applied to any two genera of lattices in the same vector space to calculate the mass of one genus knowing the mass of the other. Formulas for the numbers of maximal isotropic subspaces in a symplectic or unitary space over a finite field $\mathbb{F}_{q}$ may be found in [Taylor 1992, exercises (8.1), (10.4)].

The values for $\mathbb{F}_{q}=\mathbb{F}_{4}$ and dimensions $\leq 8$ are:

\begin{tabular}{|ccccccccc|}
\hline $\operatorname{dim}$ & 1 & 2 & 3 & 4 & 5 & 6 & 7 & 8 \\
$c_{1}$ & 1 & 3 & 9 & 27 & 297 & 891 & 38313 & 114939 \\
$c_{2}$ & - & 5 & - & 85 & - & 5525 & - & 1419925 \\
\hline
\end{tabular}

In the spirit of this proof we define a bipartite graph:

Definition 2.5. Let $n, h, s, a_{i j}, b_{j i}, M_{i}(1 \leq i \leq h)$, and $L_{j}(1 \leq j \leq s)$ be as in Proposition 2.4. Then $\Gamma_{\text {iso }}(n)$ is the labelled bipartite graph with vertices $M_{i}$ and $L_{j}$ and edges

$$
\left\{\left(M_{i}, L_{j}\right): a_{i j}>0\right\} \cup\left\{\left(L_{j}, M_{i}\right): b_{j i}>0\right\}
$$

labelled with the corresponding number $a_{i j}$ or $b_{j i}$, respectively.

Proposition 2.6. (i) $\Gamma_{\text {iso }}(n)$ is connected.

(ii) The valence of each of the vertices $M_{i}$ is $c_{1}$ and the valence of each of the vertices $L_{j}$ is $c_{2}$, where $c_{1}$ and $c_{2}$ are defined as in Proposition 2.4.

(iii) Every subgraph of $\Gamma_{\text {iso }}(n)$ satisfying $(i i)$ is the full graph $\Gamma_{\text {iso }}(n)$.
Proof. (i) Let $M$ and $M^{\prime}$ be two $\mathfrak{M}$-unimodular lattices. By [Bachoc 1995] there is a sequence of $\mathfrak{M}$-unimodular lattices $M:=M_{1}^{\prime}, \ldots, M_{k}^{\prime}=: M^{\prime}$ with $M_{i}^{\prime} /\left(M_{i}^{\prime} \cap M_{i+1}^{\prime}\right) \cong \mathfrak{M} / \mathfrak{P}(1 \leq i<k)$. For $1 \leq$ $i<k$ let $K_{i}:=\left(M_{i}^{\prime} \cap M_{i+1}^{\prime}\right)$. Then the orthogonal complement with respect to the Hermitian form $\bar{h}$ of Proposition 2.2 of $K_{i}+\mathfrak{P} M_{i}^{\prime}$ is $K_{i}^{\perp}+\mathfrak{P} M_{i}^{\prime}=$ $\mathfrak{P}\left\langle M_{i+1}^{\prime}, M_{i}^{\prime}\right\rangle+\mathfrak{P} M_{i}^{\prime}$ and contained in $K_{i}+\mathfrak{P} M_{i}^{\prime}$. Therefore $K_{i}+\mathfrak{P} M_{i}^{\prime}$ contains a maximal isotropic subspace of $M_{i}^{\prime} / \mathfrak{P} M_{i}^{\prime}$. For $1 \leq i<k$ let $L_{i}^{\prime}$ be a full preimage of a maximal isotropic subspace of $M_{i}^{\prime} / \mathfrak{P} M_{i}^{\prime}$ contained in $M_{i+1}^{\prime}$. Then $L_{1}^{\prime}, \ldots, L_{k-1}^{\prime}$ is a chain of (almost) $\mathfrak{P}$-modular lattices joining $M$ and $M^{\prime}$ in $\Gamma_{\text {iso }}(n)$.

(ii) follows from Proposition 2.4 and (iii) is an easy consequence of (i).

\section{SOME NOTATION}

In this section we give constructions for the occuring root lattices and notation for the classified lattices. The $\mathfrak{M}$-lattices of rank $n$ are understood to lie in the $\mathfrak{Q}$ vector space $\mathfrak{Q}^{n}$ endowed with the Hermitian form $h(x, y)=\sum_{i=1}^{n} x_{i} \bar{y}_{i}$. When considered as $\mathbb{Z}$-lattices, the corresponding scalar product is as defined in Section 2.

\section{Rational Root Lattices}

Let $n \geq 1$. The standard lattice $\mathbb{Z}^{n}$ is the $\mathbb{Z}$-span of an orthonormal basis of an $n$-dimensional Euclidean vector space.

Let $n \geq 1$. The root lattice $\mathbb{A}_{n}$ is defined as the $n$-dimensional sublattice

$$
\mathbb{A}_{n}:=\left\{\left(x_{0}, \ldots, x_{n}\right) \in \mathbb{Z}^{n+1}: \sum_{i=0}^{n} x_{i}=0\right\}
$$

of the standard lattice $\mathbb{Z}^{n+1}$. The discriminant group $\mathbb{A}_{n}^{\#} / \mathbb{A}_{n}$ is cyclic of order $n+1$ and generated by $\overline{p\left(\varepsilon_{0}\right)}$, where

$$
p\left(\varepsilon_{0}\right)=\frac{1}{n+1}(n,-1, \ldots,-1) \in \mathbb{A}_{n}^{\#}
$$

is the projection of the first basis vector $(1,0, \ldots, 0)$ of $\mathbb{Z}^{n+1}$ into $\mathbb{Q} \mathbb{A}_{n}$. 
For $n \geq 4$ the root lattice $\mathbb{D}_{n}$ is defined as the even sublattice of $\mathbb{Z}^{n}$,

$$
\mathbb{D}_{n}:=\left\{\left(x_{1}, \ldots, x_{n}\right) \in \mathbb{Z}^{n}: \sum_{i=1}^{n} x_{i} \in 2 \mathbb{Z}\right\} .
$$

If $n=8$, the standard lattice $\mathbb{Z}^{8}$ has an even neighbor $\mathbb{E}_{8}$ containing $\mathbb{D}_{8}$ :

$$
\mathbb{E}_{8}:=\left\langle\mathbb{D}_{8}, \frac{1}{2}(1,1,1,1,1,1,1,1)\right\rangle_{\mathbb{Z}}
$$

\section{Complex Root Lattices}

There is one infinite series of quaternionic lattices, which can be uniformly described as sublattices of $\mathfrak{M}^{n}$ [Martinet 1996]. Let $\mathfrak{I}$ be a left ideal of $\mathfrak{M}$. For $n \geq 1$ define

$$
D_{n}(\mathfrak{I}):=\left\{\left(x_{1}, \ldots, x_{n}\right) \in \mathfrak{M}^{n}: \sum_{i=0}^{n} x_{i} \in \mathfrak{I}\right\} .
$$

These quaternionic lattices are in fact scalar extensions of complex lattices, since they may be defined over any subfield of $\mathfrak{Q}$ containing generators of the left $\mathfrak{M}$-ideal $\mathfrak{I}$.

The $\mathfrak{P}$-modular $\mathfrak{M}$-lattice $D_{2}((1+i))$ is as a $\mathbb{Z}$ lattice isometric to the root lattice $\mathbb{E}_{8}$ and therefore denoted by $E_{8}$.

We additionally need one lattice defined over $\mathbb{Z}[\omega]$ where $\omega:=\frac{1}{2}(-1+\sqrt{-3})$ is a third root of unity. This root lattice is described in [Feit 1978] as an extension of $\mathbb{A}_{5}$ :

$U_{5}:=\left\langle\mathbb{A}_{5}, \frac{1-\omega}{3}\left(1, \omega, \omega^{2}, 1, \omega, \omega^{2}\right)\right\rangle_{\mathbb{Z}[\omega]} \subseteq \mathbb{Q}[\omega] \otimes \mathbb{Z}^{6}$.

The $\mathfrak{M}$-unimodular lattice $R_{24}$ as defined in [Bachoc 1995] can be constructed as $\mathfrak{M} \otimes_{\mathbb{Z}[\omega]} U_{6}$, where the root lattice $U_{6}$ is defined in [Feit 1978].

\section{Hermitian Root Lattices}

For the Hermitian root lattices, we refer to the notations of the root systems in [Cohen 1980].

The root lattice

$$
B W_{16}:=D_{4}((1+i))+\mathfrak{P}^{-1}(1,1,1,1)
$$

is an $\mathfrak{M}$-unimodular lattice spanned by the root system $S_{3}$. It is denoted by $B W_{16}$, because the corresponding $\mathbb{Z}$-lattice is the well known BarnesWall lattice $B W_{16}$ of dimension 16 .

We set

$$
S_{1}:=\left\{y \in B W_{16}: h(x, y) \in \mathfrak{P}\right\},
$$

where $x \in B W_{16}$ is any vector in $B W_{16}$ satisfying $h(x, x)=3$. The sublattice of index $B W_{16} / S_{1} \cong$ $\mathfrak{M} / \mathfrak{P}$ in $B W_{16}$ is spanned by the root system $S_{1}$.

The lattice $R_{20}$ of [Bachoc 1995] is the M-unimodular lattice spanned by the root system $U$.

Remark 3.1. Let $\Lambda$ be an (almost) $\mathfrak{P}$-modular $\mathfrak{M}$ lattice of rank $n$. If the corresponding $\mathbb{Z}$-lattice $L$ has vectors of length 2 , the $\mathfrak{M}$-lattice generated by these vectors is of the form $E_{8}^{m} \perp \mathfrak{P}^{s}$, where $E_{8}^{m}$ is an orthogonal summand of $\Lambda$ [Quebbemann 1984]. If $n \leq 8$ the vectors of length 2 in $L$ turn out to determine $\Lambda$ up to isometry.

In view of this remark let $L_{n}\left(\mathfrak{P}^{s}\right)$ denote the (almost) $\mathfrak{P}$-modular $\mathfrak{M}$-lattice of rank $n$ such that the corresponding $\mathbb{Z}$-lattice has root lattice $\mathbb{D}_{4}^{s}$.

In particular $L_{n}\left(\mathfrak{P}^{n}\right)$ is the (almost) $\mathfrak{P}$-modular lattice constructed using the indecomposable code $e_{n}$, described in [MacWilliams et al. 1978], which corresponds to a maximal isotropic subspace of $(\mathfrak{M} / \mathfrak{P})^{n}=\mathbb{F}_{4}^{n}$.

The lattice $L_{7}\left(\mathfrak{P}^{3}\right)$ is a suitable lattice containing $B W_{16} \perp \mathfrak{P}^{3}$ of index $(\mathfrak{M} / \mathfrak{P})^{3}$.

$L_{7}(\mathfrak{P})$ contains $\mathfrak{P} \perp L_{24}$ with index $\mathfrak{M} / \mathfrak{P}$, where $L_{24}$ is a maximal common $\mathfrak{M}$-sublattice of $\Lambda_{24}$ and $L_{6}\left(\mathfrak{P}^{6}\right)$.

$\Lambda_{4 n}$ denotes (almost) $\mathfrak{P}$-modular lattices such that the corresponding $\mathbb{Z}$-lattices have no vectors of length 2. For $n=6$ one gets the $\mathfrak{M}$-structure of the Leech lattice as described in [Tits 1980]. The uniqueness of this structure is proved in [Quebbemann 1984]. In [Quebbemann 1995] an integral $\mathbb{Z}$-lattice of dimension 28, determinant 4 and minimum 4 is described. As can be seen from the construction, this lattice has a structure over $\mathfrak{M}$ and hence is isometric to $\Lambda_{28}$. Compare [Nebe 1996], where the corresponding $\mathbb{Z}$-lattice is denoted by $\left[2 . J_{2}{ }^{2(2)} \mathrm{SL}_{2}(3)\right]_{28}$, and [Bacher and Venkov 1996]. 
The labels $a_{i j}$ and $b_{j i}$ of the edges in the pictures of $\Gamma_{\text {iso }}(n)$ for $n \leq 6$ are omitted. For $n<4$ these labels may easily be calculated from the table given just before Definition 2.5. For $n \geq 4$, the graphs $\Gamma_{\text {iso }}(n)$ are represented by tables. The columns of these tables correspond to the isomorphism classes of (almost) $\mathfrak{P}$-modular lattices $L_{1}, \ldots, L_{s}$, the rows to those of $\mathfrak{M}$-unimodular lattices $M_{1}, \ldots, M_{h}$. For each lattice $K$, a name and the order of its Hermitian automorphism group $U(K)$ is given. The entry $(i, j)$ of the table itself consists of the numbers $a_{i j}$ and $b_{j i}$ in the notation of Proposition 2.4, where $1 \leq i \leq h$ and $1 \leq j \leq s$.

\section{RESULTS FOR RANK 1 TO 7}

Tables 1 and 2 present the graphs $\Gamma_{\text {iso }}(n)$ for $n \leq 7$. The occuring $\mathfrak{M}$-unimodular lattices have already been determined in [Bachoc 1995], from which we also borrow some notation.
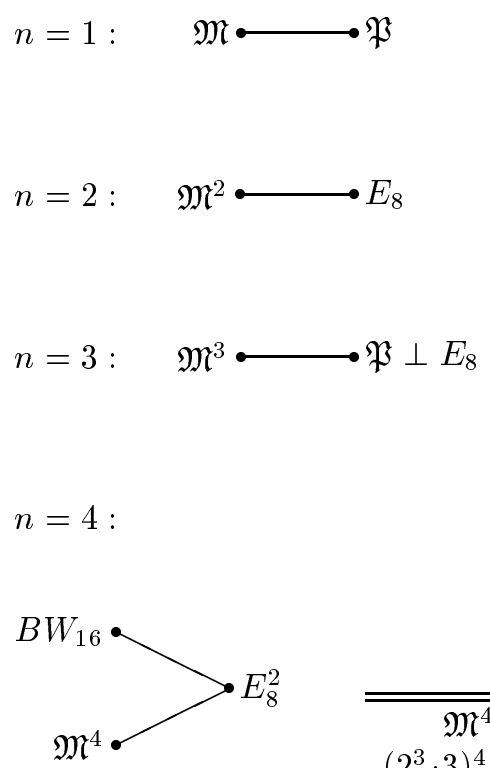

$$
n=5 \text { : }
$$

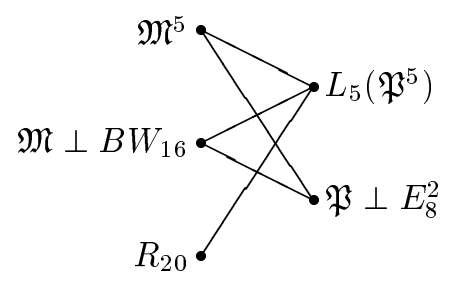

$n=6:$

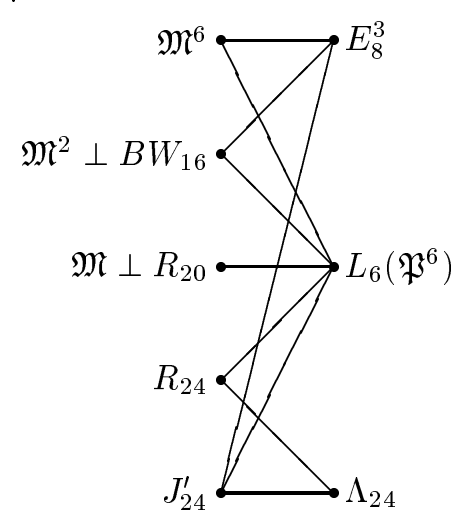

\begin{tabular}{c||r|r} 
& $L_{5}\left(\mathfrak{P}^{5}\right)$ & $\mathfrak{P} \perp E_{8}^{2}$ \\
& $2^{17} \cdot 3^{2} \cdot 5$ & $2^{18} \cdot 3^{3} \cdot 5^{2}$ \\
\hline \hline $\mathfrak{M}^{5}$ & 162 & 135 \\
$\left(2^{3} \cdot 3\right)^{5} \cdot(5 !)$ & 1 & 25 \\
\hline $\mathfrak{M} \perp B W_{16}$ & 270 & 27 \\
$2^{16} \cdot 3^{5} \cdot 5$ & 20 & 60 \\
\hline$R_{20}$ & 297 & \\
$2^{11} \cdot 3^{5} \cdot 5 \cdot 11$ & 64 &
\end{tabular}

\begin{tabular}{c||r|r|r} 
& $E_{8}^{3}$ & $L_{6}\left(\mathfrak{P}^{6}\right)$ & $\Lambda_{24}$ \\
& $\left(2^{7} \cdot 3 \cdot 5\right)^{3} \cdot 6$ & $2^{21} \cdot 3^{3} \cdot 5$ & $2^{13} \cdot 3^{3} \cdot 5^{2} \cdot 7 \cdot 13$ \\
\hline \hline $\mathfrak{M}^{6}$ & 405 & 486 & \\
$\left(2^{3} \cdot 3\right)^{6} \cdot(6 !)$ & 125 & 1 & \\
\hline $\mathfrak{M}^{2} \perp B W_{16}$ & 81 & 810 & \\
$2^{20} \cdot 3^{6} \cdot 5$ & 900 & 60 & \\
\hline $\mathfrak{M} \perp R_{20}$ & & 891 & \\
$2^{14} \cdot 3^{6} \cdot 5 \cdot 11$ & & 384 & 324 \\
\hline$R_{24}$ & & 567 & 4160 \\
$2^{9} \cdot 3^{7} \cdot 5 \cdot 7$ & & 4096 & 384 \\
\hline$J_{24}^{\prime}$ & 15 & 492 & 1365 \\
$2^{16} \cdot 3 \cdot(6 !)$ & 4500 & 984 &
\end{tabular}

TABLE 1. The graph $\Gamma_{\text {iso }}(n)$ and information about its edges, for $n=1, \ldots, 6$. 


\begin{tabular}{|c|c|c|c|c|c|c|c|c|}
\hline & $\begin{array}{r}E_{8}^{3} \perp \mathfrak{P} \\
2^{25} \cdot 3^{5} \cdot 5^{3}\end{array}$ & $\begin{array}{r}L_{6}\left(\mathfrak{P}^{6}\right) \perp \mathfrak{P} \\
2^{24} \cdot 3^{4} \cdot 5\end{array}$ & $\begin{array}{r}\Lambda_{24} \perp \mathfrak{P} \\
2^{16} \cdot 3^{4} \cdot 5^{2} \cdot 7 \cdot 13\end{array}$ & $\begin{array}{r}L_{5}\left(\mathfrak{P}^{5}\right) \perp E_{8} \\
2^{24} \cdot 3^{3} \cdot 5^{2}\end{array}$ & $\begin{array}{r}L_{7}\left(\mathfrak{P}^{7}\right) \\
2^{24} \cdot 3^{2} \cdot 7\end{array}$ & $\begin{array}{r}L_{7}\left(\mathfrak{P}^{3}\right) \\
2^{17} \cdot 3^{4}\end{array}$ & $\begin{array}{r}L_{7}(\mathfrak{P}) \\
2^{16} \cdot 3^{2} \cdot 5\end{array}$ & $\begin{array}{r}\Lambda_{28} \\
2^{8} \cdot 3^{3} \cdot 5^{2} \cdot 7\end{array}$ \\
\hline $\begin{array}{c}\mathfrak{M}^{7} \\
\left(2^{3} \cdot 3\right)^{7} \cdot(7 !) \\
\end{array}$ & $\begin{array}{r}2835 \\
125 \\
\end{array}$ & $\begin{array}{r}3402 \\
1 \\
\end{array}$ & & $\begin{array}{r}10206 \\
5 \\
\end{array}$ & $\begin{array}{r}21870 \\
1 \\
\end{array}$ & & & \\
\hline $\begin{array}{c}\mathfrak{M}^{3} \perp B W_{16} \\
2^{23} \cdot 3^{8} \cdot 5 \\
\end{array}$ & $\begin{array}{l}243 \\
900 \\
\end{array}$ & $\begin{array}{r}2430 \\
60 \\
\end{array}$ & & $\begin{array}{r}2430 \\
100 \\
\end{array}$ & $\begin{array}{r}7290 \\
28 \\
\end{array}$ & $\begin{array}{r}25920 \\
1 \\
\end{array}$ & & \\
\hline $\begin{array}{c}\mathfrak{M}^{2} \perp R_{20} \\
2^{18} \cdot 3^{7} \cdot 5 \cdot 11 \\
\end{array}$ & & $\begin{array}{r}1782 \\
384 \\
\end{array}$ & & $\begin{array}{l}891 \\
320 \\
\end{array}$ & & $\begin{array}{r}35640 \\
12 \\
\end{array}$ & & \\
\hline $\begin{array}{c}\mathfrak{M} \perp R_{24} \\
2^{12} \cdot 3^{8} \cdot 5 \cdot 7 \\
\end{array}$ & & $\begin{array}{r}567 \\
4096 \\
\end{array}$ & $\begin{array}{r}324 \\
4160 \\
\end{array}$ & & & $\begin{array}{r}17010 \\
192 \\
\end{array}$ & $\begin{array}{r}20412 \\
64 \\
\end{array}$ & \\
\hline $\begin{array}{c}\mathfrak{M} \perp J_{24}^{\prime} \\
2^{19} \cdot 3^{2} \cdot(6 !) \\
\end{array}$ & $\begin{array}{r}15 \\
4500 \\
\end{array}$ & $\begin{array}{l}492 \\
984 \\
\end{array}$ & $\begin{array}{r}384 \\
1365 \\
\end{array}$ & $\begin{array}{r}90 \\
300 \\
\end{array}$ & $\begin{array}{r}1620 \\
504 \\
\end{array}$ & $\begin{array}{r}11520 \\
36 \\
\end{array}$ & $\begin{array}{r}24192 \\
21 \\
\end{array}$ & \\
\hline $\begin{array}{c}R_{28} \\
2^{12} \cdot 3^{5} \cdot 5 \cdot 7 \\
\end{array}$ & & & & & $\begin{array}{r}135 \\
4096 \\
\end{array}$ & $\begin{array}{r}1890 \\
576 \\
\end{array}$ & $\begin{array}{r}12096 \\
1024 \\
\end{array}$ & $\begin{array}{r}24192 \\
840 \\
\end{array}$ \\
\hline $\begin{array}{c}R_{28}^{\prime} \\
2^{18} \cdot 3^{5} \\
\end{array}$ & & & & $\begin{array}{r}27 \\
4800 \\
\end{array}$ & $\begin{array}{r}54 \\
896 \\
\end{array}$ & $\begin{array}{r}3672 \\
612 \\
\end{array}$ & $\begin{array}{r}6912 \\
320 \\
\end{array}$ & $\begin{array}{r}27648 \\
525 \\
\end{array}$ \\
\hline $\begin{array}{c}R_{28}^{\prime \prime} \\
2 \cdot 3^{6} \cdot(7 !)\end{array}$ & & & & & & $\begin{array}{l}2835 \\
4096\end{array}$ & $\begin{array}{r}10206 \\
4096\end{array}$ & $\begin{array}{r}25272 \\
4160\end{array}$ \\
\hline
\end{tabular}

TABLE 2. Information about the edges of $\Gamma_{\text {iso }}(7)$.

\section{THE LATTICES OF RANK 8}

In this section we present the main result, the classification of the $\mathfrak{M}$-unimodular and the $\mathfrak{P}$-modular lattices of rank 8. For the matrix groups, the notation is borrowed from [Nebe and Plesken 1995; Nebe $\geq 1997]$. In particular we call a matrix group absolutely irreducible if the $\mathbb{Q}$-algebra generated by the matrices in the group is the full matrix algebra.

Theorem 5.1. There are 11 isometry classes of $\mathfrak{P}$ modular lattices of rank 8. Seven of them yield unimodular $\mathbb{Z}$-lattices that contain roots; they may be distinguished by their root lattices, which are $\mathbb{D}_{4}$, $\mathbb{D}_{4}^{2}, \mathbb{D}_{4}^{4}, \mathbb{D}_{4}^{8}, \mathbb{E}_{8}, \mathbb{E}_{8} \perp \mathbb{D}_{4}^{6}$, and $\mathbb{E}_{8}^{4}$. The other four lattices can be distinguished by means of their Hermitian automorphism groups (which have been investigated using MAGMA.)

(i) $U\left(B W_{32}\right)=\left(Q_{8} \otimes D_{8} \otimes D_{8} \otimes D_{8}\right) \cdot O_{8}^{-}(2)$ is an $a b$ solutely irreducible maximal finite subgroup of $\mathrm{GL}_{8}(\mathfrak{Q})$. The automorphism group of the corresponding unimodular $\mathbb{Z}$-lattice is $\operatorname{Aut}\left(B W_{32}\right)=$ $\left(D_{8} \otimes D_{8} \otimes D_{8} \otimes D_{8} \otimes D_{8}\right) . O_{10}^{+}(2)$ and an absolutely irreducible maximal finite subgroup of $\mathrm{GL}_{32}(\mathbb{Q})$. (ii) $U\left(\Lambda_{32}^{\prime \prime}\right)=2_{-}^{1+6} \cdot O_{6}^{-}(2) \wedge 2_{-}^{1+6} O_{6}^{-}(2)$ is the $s u b$ direct product of two groups $2_{-}^{1+6} . O_{6}^{-}(2)=$ $\left(Q_{8} \otimes D_{8} \otimes D_{8}\right) . O_{6}^{-}(2)=U\left(B W_{16}\right)$ amalgamated of the common factor group $\mathrm{O}_{6}^{-}(2)$. This group is a reducible subgroup of $\mathrm{GL}_{8}(\mathfrak{Q})$. The corresponding unimodular $\mathbb{Z}$-lattice is isometric to $B W_{32}$.

(iii) $U\left(\Lambda_{32}\right)=\left(C_{4} \otimes D_{8} \otimes D_{8} \otimes D_{8}\right) .\left(U_{3}(3)\right.$.2) is an absolutely irreducible subgroup of $U\left(B W_{32}\right)$. The automorphism group of the corresponding unimodular $\mathbb{Z}$-lattice is $\operatorname{Aut}\left(\Lambda_{32}\right)=\left(C_{4} \otimes D_{8} \otimes D_{8} \otimes\right.$ $\left.D_{8}\right) \cdot\left(S_{3} \times U_{3}(3) .2\right)$ and an absolutely irreducible subgroup of $\operatorname{Aut}\left(B W_{32}\right)$.

(iv) $U\left(\Lambda_{32}^{\prime}\right)=\left(\mathrm{SL}_{2}(5) \circ \mathrm{SL}_{2}(5) \otimes \mathrm{SL}_{2}(5)\right): S_{3}$ is an absolutely irreducible maximal finite subgroup of $\mathrm{GL}_{4}(\mathfrak{Q} \otimes \mathbb{Q}[\sqrt{5}])$. The automorphism group of the corresponding unimodular $\mathbb{Z}$-lattice is

$$
\begin{aligned}
& \operatorname{Aut}\left(\Lambda_{32}^{\prime}\right)= \\
& \quad\left(\left(\mathrm{SL}_{2}(5) \circ \mathrm{SL}_{2}(5) \underset{\sqrt{5}}{\otimes} \mathrm{SL}_{2}(5) \circ \mathrm{SL}_{2}(5)\right) .2\right): S_{4}
\end{aligned}
$$

and an absolutely irreducible maximal finite subgroup of $\mathrm{GL}_{32}(\mathbb{Q})$. 


\begin{tabular}{|c|c|c|c|}
\hline root lattice $R$ & construction of $M$ & {$[M: R]$} & $\left|R_{4}\right|$ \\
\hline$D_{8}(1+i)$ & $\frac{1+i}{2}(1,1,1,1,1,1,1,1)$ & $2^{2}$ & $24 \cdot 232$ \\
\hline$D_{8}(1-\omega)$ & $\frac{1-\omega}{3}(1,1+\alpha, 1,1+\alpha, 1,1+\alpha, 1,1+\alpha)$, for $\alpha=i(1-\omega)$ & $3^{2}$ & $24 \cdot 84$ \\
\hline$D_{4}(1+i)^{2}$ & $\begin{array}{l}\frac{1-i}{2}(1,1,1,1,0,0,0,(1+i)) \\
\frac{1-i}{2}(0,0,0,(1+i), 1,1,1,1)\end{array}$ & $2^{4}$ & $24 \cdot 104$ \\
\hline$S_{1}^{2}$ & $\begin{array}{l}(x,-x) \\
\frac{1+i}{2}(x, x)\end{array}$ & $2^{4}$ & $24 \cdot 72$ \\
\hline $\mathfrak{M} \otimes \mathbb{D}_{8}$ & $\frac{1}{2}(1,1,1,1,1,1,1,3+i)$ & $2^{4}$ & $24 \cdot 56$ \\
\hline$D_{3}(1+i) \perp \mathfrak{M} \underset{\mathbb{Z}[\omega]}{\otimes} U_{5}$ & $\left(\frac{1+i}{2}(1,1,1),(1-\omega) p\left(\varepsilon_{0}\right)\right)$ & $2^{4}$ & $24 \cdot 72$ \\
\hline$D_{2}(1+i) \perp \mathfrak{M} \otimes \mathbb{D}_{6}$ & $\begin{array}{l}\left((1,0), \frac{1}{2}\left(\omega-\omega^{2}, 1,1,1,1,1\right)\right) \\
\left(\frac{1+i}{2}(1,1),(0,1+i, 0,0,0,0)\right)\end{array}$ & $2^{6}$ & $24 \cdot 40$ \\
\hline$D_{4}(1-\omega)^{2}$ & $\begin{array}{l}\left((1,1,1,1),\left(\frac{1-\omega}{3}+i\right)(1,1,1,1)\right) \\
\left(\left(\frac{-2-\omega}{3}+i\right)(1,1,1,1),(1,1,1,1)\right)\end{array}$ & $3^{4}$ & $24 \cdot 36$ \\
\hline $\mathfrak{M} \otimes \mathbb{A}_{8}$ & $(-2+3 i-4 \omega) p\left(\varepsilon_{0}\right)$ & $9^{2}$ & $24 \cdot 36$ \\
\hline$D_{2}(1+i)^{4}$ & $\begin{array}{l}\frac{1-i}{2}(1,1,1,1,0,0,0,(1+i)) \\
\frac{1-i}{2}(0,0,1,1,1,1+(1+i) \omega, 0,(1+i) \omega) \\
\frac{1-i}{2}((1+i), 0,0,0,1,1,1,1) \\
(0,1,0,1,0,1,0,1,0,1)\end{array}$ & $2^{8}$ & $24 \cdot 40$ \\
\hline $\mathfrak{M} \otimes \mathbb{D}_{4}^{2}$ & $\begin{array}{l}\frac{1}{2}(2 i, 0,0,0,1+2 i, 1,1,1) \\
\frac{1}{2}(1+2 j, 1,1,1,2 i, 0,0,0)\end{array}$ & $2^{8}$ & $24 \cdot 24$ \\
\hline$D_{3}(1-\omega) \perp \mathfrak{M} \otimes \mathbb{A}_{5}$ & $\begin{array}{l}\left((1,0,0), \frac{1-\omega}{3}\left(1, \omega, \omega^{2}, 1, \omega, \omega^{2}\right)\right) \\
(1+i)(i+2 j-\omega)\left(\frac{1-\omega}{3}(1,1,1), p\left(\varepsilon_{0}\right)\right)\end{array}$ & $3^{4} \cdot 2^{2}$ & $24 \cdot 24$ \\
\hline $\mathfrak{M} \otimes \mathbb{A}_{4}^{2}$ & $\begin{array}{l}(1+2 i)\left(p\left(\varepsilon_{0}\right), p\left(\varepsilon_{0}\right)\right) \\
(1+2 i) \omega\left(p\left(\varepsilon_{0}\right),-p\left(\varepsilon_{0}\right)\right)\end{array}$ & $5^{4}$ & $24 \cdot 20$ \\
\hline$D_{1}(1+i)^{8}$ & $\begin{array}{l}\frac{1-i}{2}(1,1,1,1,1+i, 0,0,0) \\
\frac{1-i}{2}(1+i, 0,0,0,1,1,1,1) \\
\frac{1-i}{2}(0,1, \omega, \bar{\omega}, 0,1, \omega, \bar{\omega}) \\
(0,0,1,1,0,0,1,1) \\
(0,0,0,0,0,1, \bar{\omega}, \omega)\end{array}$ & $2^{16}$ & $24 \cdot 8$ \\
\hline
\end{tabular}

TABLE 3. Information about rank- 8 lattices. The first column contains the root lattice $R$ as described in Section 3 . The $\mathfrak{M}$-unimodular lattice $M$ is generated by $R$ and the vectors given in the second column. The last column displays the number of roots in $M$. The graph $\Gamma_{\text {iso }}(8)$ is encoded in Table 4 . The indecomposable $\mathfrak{M}$-unimodular lattices are denoted by their Hermitian root systems. We follow the notations of Section 3 . Note that the last lattice in the table is the lattice $\tilde{J}_{4}^{8}$ of [Bachoc 1995]. 


\begin{tabular}{|c|c|c|c|c|c|c|}
\hline & & $\begin{array}{c}\Lambda_{32} \\
2^{14} \cdot 3^{3} \cdot 7\end{array}$ & $\begin{array}{c}\Lambda_{32}^{\prime} \\
2^{8} \cdot 3^{4} \cdot 5^{3}\end{array}$ & $\begin{array}{c}\Lambda_{32}^{\prime \prime} \\
2^{20} \cdot 3^{4} \cdot 5\end{array}$ & $\begin{array}{c}B W_{32} \\
2^{21} \cdot 3^{4} \cdot 5 \cdot 7 \cdot 17\end{array}$ & $\begin{array}{c}L_{8}(\mathfrak{P}) \\
2^{11} \cdot 3^{3} \cdot 5^{2} \cdot 7\end{array}$ \\
\hline $\mathfrak{M}^{8}$ & $\left(2^{3} \cdot 3\right)^{8} \cdot(8 !)$ & & & & & \\
\hline $\mathfrak{M}^{4} \perp B W_{16}$ & $2^{28} \cdot 3^{9} \cdot 5$ & & & & & \\
\hline$B W_{16}^{2}$ & $2^{27} \cdot 3^{8} \cdot 5^{2}$ & & & 51840 & 25920 & \\
\hline $\mathfrak{M}^{3} \perp R_{20}$ & $2^{20} \cdot 3^{9} \cdot 5 \cdot 11$ & & & & & \\
\hline $\mathfrak{M}^{2} \perp R_{24}$ & $2^{16} \cdot 3^{9} \cdot 5 \cdot 7$ & & & & & \\
\hline $\mathfrak{M}^{2} \perp J_{24}^{\prime}$ & $2^{27} \cdot 3^{5} \cdot 5$ & & & & & \\
\hline $\mathfrak{M} \perp R_{28}$ & $2^{15} \cdot 3^{6} \cdot 5 \cdot 7$ & & & & & 72576 \\
\hline $\mathfrak{M} \perp R_{28}^{\prime}$ & $2^{21} \cdot 3^{6}$ & & & & & 82944 \\
\hline $\mathfrak{M} \perp R_{28}^{\prime \prime}$ & $2^{8} \cdot 3^{9} \cdot 5 \cdot 7$ & & & & & 75816 \\
\hline $\mathfrak{M} \otimes \mathbb{E}_{8}$ & $2^{16} \cdot 3^{6} \cdot 5^{2} \cdot 7$ & & 48384 & 18900 & 3264 & \\
\hline$D_{8}(1+i)$ & $2^{29} \cdot 3^{3} \cdot 5 \cdot 7$ & & & 53760 & 3840 & \\
\hline$D_{8}(1-\omega)$ & $2^{8} \cdot 3^{9} \cdot 5 \cdot 7$ & $65610 \quad 1152$ & & & & 40824 \\
\hline$D_{4}(1+i)^{2}$ & $2^{27} \cdot 3^{3}$ & 73728 & & 10368 & $576 \quad 16065$ & \\
\hline$S_{1}^{2}$ & $2^{19} \cdot 3^{7}$ & 20736 & 55296 & 7344 & $216 \quad 19040$ & \\
\hline $\mathfrak{M} \otimes \mathbb{D}_{8}$ & $2^{16} \cdot 3^{2} \cdot 5 \cdot 7$ & $30720 \quad 4608$ & $\begin{array}{ll}48384 & 6075\end{array}$ & $2100 \quad 43200$ & $\begin{array}{ll}30 & 146880\end{array}$ & 21504 \\
\hline$\overline{D_{3}(1+i) \perp \mathfrak{M} \underset{\mathbb{Z}[\omega]}{\otimes} U_{5}}$ & $2^{15} \cdot 3^{6} \cdot 5$ & $\begin{array}{ll}77760 \quad 2016 \\
\end{array}$ & & & & 25920 \\
\hline$D_{2}(1+i) \perp \mathfrak{M} \otimes \mathbb{D}_{6}$ & $2^{16} \cdot 3^{3} \cdot 5$ & $\begin{array}{ll}40320 & 14112\end{array}$ & $49152 \quad 14400$ & $960 \quad 46080$ & & $17280 \quad 18900$ \\
\hline$D_{4}(1-\omega)^{2}$ & $2^{8} \cdot 3^{8}$ & $\begin{array}{ll}38394 & 70784\end{array}$ & $51840 \quad 80000$ & $648 \quad 163840$ & & 17496100800 \\
\hline $\mathfrak{M} \otimes \mathbb{A}_{8}$ & $2^{8} \cdot 3^{4} \cdot 5 \cdot 7$ & 41850178560 & $48384 \quad 172800$ & & & 19224256320 \\
\hline$D_{2}(1+i)^{4}$ & $2^{24} \cdot 3^{2} \cdot 5$ & $30720 \quad 126$ & $65536 \quad 225$ & $3712 \quad 2088$ & $256 \quad 34272$ & \\
\hline $\mathfrak{M} \otimes \mathbb{D}_{4}^{2}$ & $2^{15} \cdot 3^{3}$ & 42624149184 & 54528159750 & 420201600 & $\begin{array}{ll}6 & 685440\end{array}$ & 13824151200 \\
\hline$D_{3}(1-\omega) \perp \mathfrak{M} \otimes \mathbb{A}_{5}$ & $2^{8} \cdot 3^{6} \cdot 5$ & 45360150528 & 51840144000 & & & 14904154560 \\
\hline $\mathfrak{M} \otimes \mathbb{A}_{4}^{2}$ & $2^{8} \cdot 3^{3} \cdot 5^{2}$ & 45450814464 & 54144812160 & 360884736 & & 12600705600 \\
\hline$D_{1}(1+i)^{8}$ & $2^{19} \cdot 3^{2}$ & $52224 \quad 34272$ & $55296 \quad 30375$ & $816 \quad 73440$ & $24 \quad 514080$ & $\begin{array}{ll}6144 & 12600\end{array}$ \\
\hline
\end{tabular}

TABLE 4. Information about the graph $\Gamma_{\text {iso }}(8)$. The organization is as in Tables 1 and 2 , except that the numbers $a_{i j}$ and $b_{i j}$ in each cell are given side by side. The table is continued on the next page.

Remark 5.2. Up to isometry there are three extremal even unimodular $\mathbb{Z}$-lattices having a structure over $\mathfrak{M}$. The $\mathbb{Z}$-lattices corresponding to $\Lambda_{32}^{\prime \prime}$ and $B W_{32}$ are both isometric to the Barnes-Wall lattice of dimension 32. In [Koch and Venkov 1989] an invariant called the "Nachbardefekt" of an even unimodular lattice without roots is defined as the minimal corank of the root systems of its neighbor lattices.
The 15 extremal even unimodular lattices of Nachbardefekt $\leq 8$ are classified in [Koch and Venkov 1989; [1991]; Koch and Nebe 1993; Nebe 1990]. The $\mathbb{Z}$-lattice $B W_{32}$ is isometric to one of the 5 lattices of Nachbardefekt 0 . A comparison of the orders of the automorphism groups shows that the other two lattices $\Lambda_{32}$ and $\Lambda_{32}^{\prime}$ are not isometric to one of these 15 lattices. 


\begin{tabular}{|c|c|c|c|c|c|c|}
\hline & $\begin{array}{c}L_{8}\left(\mathfrak{P}^{2}\right) \\
2^{20} \cdot 3^{2} \cdot 5\end{array}$ & $\begin{array}{c}L_{8}\left(\mathfrak{P}^{4}\right) \\
2^{22} \cdot 3^{4}\end{array}$ & $\begin{array}{c}L_{8}\left(\mathfrak{P}^{8}\right) \\
2^{30} \cdot 3^{2} \cdot 7\end{array}$ & $\begin{array}{c}E_{8} \perp \Lambda_{24} \\
2^{20} \cdot 3^{4} \cdot 5^{3} \cdot 7 \cdot 13\end{array}$ & $\begin{array}{c}E_{8} \perp L_{6}\left(\mathfrak{P}^{6}\right) \\
2^{28} \cdot 3^{4} \cdot 5^{2}\end{array}$ & $\begin{array}{c}E_{8}^{4} \\
2^{31} \cdot 3^{5} \cdot 5^{4}\end{array}$ \\
\hline $\mathfrak{M}^{8}$ & & & 65610 & & 40824 & 8505 \\
\hline $\mathfrak{M}^{4} \perp B W_{16}$ & & 77760 & 21870 & & 14580 & $\begin{array}{ll}729 \quad 9000\end{array}$ \\
\hline$B W_{16}^{2}$ & & & 36450 & & & $729 \quad 10800$ \\
\hline $\mathfrak{M}^{3} \perp R_{20}$ & & 106920 & & & $\begin{array}{ll}8019 & 1920\end{array}$ & \\
\hline $\mathfrak{M}^{2} \perp R_{24}$ & 61236 & 51030 & & 20800 & $\begin{array}{ll}1701 \quad 20480 \\
\end{array}$ & \\
\hline $\mathfrak{M}^{2} \perp J_{24}^{\prime}$ & 72576 & 34560 & $4860 \quad 2016$ & 6825 & $\begin{array}{ll}1746 & 5820\end{array}$ & $45 \quad 90000$ \\
\hline $\mathfrak{M} \perp R_{28}$ & $36288 \quad 2048$ & $5670 \quad 2304$ & $405 \quad 32768$ & & & \\
\hline $\mathfrak{M} \perp R_{28}^{\prime}$ & $20736 \quad 640$ & $11016 \quad 2448$ & $162 \quad 7168$ & & 8128800 & \\
\hline $\mathfrak{M} \perp R_{28}^{\prime \prime}$ & $\begin{array}{ll}30618 \quad 8192\end{array}$ & $8505 \quad 16384$ & & & & \\
\hline $\mathfrak{M} \otimes \mathbb{E}_{8}$ & $45360 \quad 256$ & & $2025 \quad 16384$ & & & \\
\hline$D_{8}(1+i)$ & 43008 & & $13890 \quad 1852$ & & 336 & $\overline{105 \quad 67500}$ \\
\hline$D_{8}(1-\omega)$ & & $8505 \quad 16384$ & & & & \\
\hline$D_{4}(1+i)^{2}$ & $18432 \quad 240$ & $10368 \quad 972$ & $1170 \quad 21840$ & & $\begin{array}{ll}288 \quad 43200 \\
\end{array}$ & 9810000 \\
\hline$S_{1}^{2}$ & $31104 \quad 1280$ & & $243 \quad 14336$ & & & \\
\hline $\mathfrak{M} \otimes \mathbb{D}_{8}$ & $10416 \quad 23808$ & $1680 \quad 27648$ & 105344064 & & & \\
\hline$D_{3}(1+i) \perp \mathfrak{M}_{\mathbb{Z}[\omega]}^{\otimes} U_{5}$ & $\begin{array}{ll}3888 & 1536\end{array}$ & $7290 \quad 20736$ & & & 81368640 & \\
\hline$D_{2}(1+i) \perp \mathfrak{M} \otimes \mathbb{D}_{6}$ & $5460 \quad 29120$ & $1710 \quad 65664$ & 30229376 & $12 \quad 1310400$ & 15921600 & \\
\hline$D_{4}(1-\omega)^{2}$ & $5832 \quad 163840$ & 729147456 & & & & \\
\hline $\mathfrak{M} \otimes \mathbb{A}_{8}$ & 4536294912 & 945442368 & & & & \\
\hline$D_{2}(1+i)^{4}$ & $12480 \quad 780$ & $1920 \quad 864$ & $\begin{array}{ll}210 & 18816 \\
\end{array}$ & 81900 & $\begin{array}{ll}40 & 28800\end{array}$ & 1432000 \\
\hline $\mathfrak{M} \otimes \mathbb{D}_{4}^{2}$ & 3312176640 & $\begin{array}{ll}216 & 82944 \\
\end{array}$ & 9688128 & & & \\
\hline$D_{3}(1-\omega) \perp \mathfrak{M} \otimes \mathbb{A}_{5}$ & 2430122880 & 405147456 & & & & \\
\hline $\mathfrak{M} \otimes \mathbb{A}_{4}^{2}$ & 2160589824 & 225442368 & & & & \\
\hline$D_{1}(1+i)^{8}$ & $\begin{array}{ll}384 & 3840\end{array}$ & $\begin{array}{ll}48 & 3456\end{array}$ & \begin{tabular}{|ll}
3 & 43008
\end{tabular} & & & \\
\hline
\end{tabular}

TABLE 4 (continued). Information about the graph $\Gamma_{\text {iso }}(8)$.

Theorem 5.3. There are 24 isometry classes of $\mathfrak{M}$ unimodular lattices of rank 8, fifteen of which consist of indecomposable lattices. These fifteen lattices may be distinguished via their Hermitian root system which is in all cases of full rank. In particular, there is no extremal 2-modular integral lattice of dimension 32 having a structure as an $\mathfrak{M}$ unimodular lattice.
A description of the $\mathfrak{M}$-unimodular lattices of dimension 32 may be obtained using their Hermitian root systems as given in Section 3, and is encoded in Tables 3 and 4.

The method used to find representatives for the isometry classes of the lattices in the two genera of $\mathfrak{M}$-unimodular and $\mathfrak{P}$-modular lattices can be described as follows: 
(1) Starting with decomposable $\mathfrak{M}$-unimodular lattices $M$, we calculate the orbits of $U(M)$ on the 114939 maximal isotropic subspaces of the Hermitian $\mathbb{F}_{4}$ vector space $M / \mathfrak{P} M$ and the corresponding $\mathfrak{P}$-modular lattices $L$ as full preimages of representatives of the orbits.

(2) For the lattices $L$ found in (1) we check whether $L$ is already known. If not, we determine $U(L)$ with a computer program described in [Plesken and Souvignier $\geq 1997$.

(3) For the lattices $L$ found in (1) we compute the number of sublattices $M^{\prime} \leq L^{*}$, which are isometric to $M$ using Equation (2.1) on page 154 .

(4) When all known $\mathfrak{M}$-unimodular lattices $M$ are processed in this way, we look for new $\mathfrak{M}$-unimodular lattices as full preimages of maximal isotropic subspaces of $L^{*} / L$, where $L$ is one of the known $\mathfrak{P}$-modular lattices.

Remark 5.4. For the computation of $U(L)$ in (2) it is helpful to know a subgroup of $U(L)$ which is obtained computing some elements of $U(M)$ stabilizing the maximal isotropic subspace $L / \mathfrak{P} M$. An analogous remark applies to (4).

Remark 5.5. To check whether $L$ is already known, it suffices in most cases to compute the number of roots in $L$. To prove the completeness of the list of $\mathfrak{P}$-modular and $\mathfrak{M}$-unimodular lattices we use the mass formula. An analogous remark applies to (4).

Remark 5.6. Since one knows a priori the number of maximal isotropic subspaces of $L^{*} / L$ yielding known $\mathfrak{M}$-unimodular lattices by step (3), one can choose $L$ such that one has a good chance to find new $\mathfrak{M}$-unimodular lattices.

\section{REFERENCES}

[Bacher and Venkov 1996] R. Bacher and B. B. Venkov, "Réseaux entiers unimodulaires sans racine en dimension 27 et 28", preprint, Institut Fourier, Grenoble, 1996.

[Bachoc 1995] C. Bachoc, "Voisinage au sens de Kneser pour les réseaux quaternioniens", Comment. Math. Helv. 70:3 (1995), 350-374.
[Cohen 1980] A. M. Cohen, "Finite quaternionic reflection groups", J. Algebra 64:2 (1980), 293-324.

[Feit 1978] W. Feit, "Some lattices over $\mathbb{Q}(\sqrt{ }-3)$ ", J. Algebra 52:1 (1978), 248-263.

[Hashimoto 1980] K.-i. Hashimoto, "On Brandt matrices associated with the positive definite quaternion Hermitian forms", J. Fac. Sci. Univ. Tokyo Sect. IA Math. 27:1 (1980), 227-245.

[Kneser 1957] M. Kneser, "Klassenzahlen definiter quadratischer Formen", Arch. Math. 8 (1957), 241250 .

[Koch and Nebe 1993] H. Koch and G. Nebe, "Extremal even unimodular lattices of rank 32 and related codes", Math. Nachr. 161 (1993), 309-319.

[Koch and Venkov 1989] H. Koch and B. B. Venkov, "Über ganzzahlige unimodulare euklidische Gitter", J. Reine Angew. Math. 398 (1989), 144-168.

[Koch and Venkov 1991] H. Koch and B. B. Venkov, "Über gerade unimodulare Gitter der Dimension 32, III", Math. Nachr. 152 (1991), 191-213.

[MacWilliams et al. 1978] F. J. MacWilliams, A. M. Odlyzko, N. J. A. Sloane, and H. N. Ward, "Selfdual codes over GF(4)", J. Combin. Theory Ser. A 25:3 (1978), 288-318.

[Martinet 1996] J. Martinet, Les réseaux parfaits des espaces euclidiens, Mathématiques, Masson, Paris, 1996.

[Nebe 1990] G. Nebe, Wiedererkennung von Gittern, Diplomarbeit, Lehrstuhl B für Mathematik, RWTH Aachen, 1990.

[Nebe 1996] G. Nebe, "Finite subgroups of $\mathrm{GL}_{n}(\mathbb{Q})$ for $25 \leq n \leq 31$ ", Comm. Algebra 24:7 (1996), 23412397.

[Nebe $\geq 1997] \quad$ G. Nebe, "Finite quaternionic matrix groups". See http://samuel.math.rwth-aachen. de/ LBFM/gabi. Submitted.

[Nebe and Plesken 1995] G. Nebe and W. Plesken, "Finite rational matrix groups", Mem. Amer. Math. Soc. 116:556 (1995), viii+144.

[Plesken and Souvignier $\geq 1997]$ W. Plesken and B. Souvignier, "Computing isometries of lattices". To appear in J. Symbolic Comput. 
[Quebbemann 1984] H.-G. Quebbemann, "An application of Siegel's formula over quaternion orders", Mathematika 31:1 (1984), 12-16.

[Quebbemann 1995] H.-G. Quebbemann, "Modular lattices in Euclidean spaces", J. Number Theory 54:2 (1995), 190-202.
[Taylor 1992] D. E. Taylor, The geometry of the classical groups, Sigma Series in Pure Mathematics, Heldermann Verlag, Berlin, 1992.

[Tits 1980] J. Tits, "Four presentations of Leech's lattice", pp. 303-307 in Finite simple groups, II (Durham, 1978), edited by M. J. Collins, Academic Press, London and New York, 1980.

Christine Bachoc, A2X Bordeaux, Université de Bordeaux I, 351 cours de la Libération, 33405 Talence, France (bachoc@math.u-bordeaux.fr)

Gabriele Nebe, Lehrstuhl B für Mathematik, RWTH Aachen, Templergraben 64, 52062 Aachen, Germany (gabi@willi.math.rwth-aachen.de) 